\title{
Transparent and conducting electrodes for organic electronics from reduced graphene oxide
}

\author{
Goki Eda, ${ }^{1, a)}$ Yun-Yue Lin, ${ }^{2}$ Steve Miller, ${ }^{1}$ Chun-Wei Chen, ${ }^{2, a)}$ Wei-Fang Su, ${ }^{2}$ and \\ Manish Chhowalla ${ }^{1, a)}$ \\ ${ }^{1}$ Materials Science and Engineering, Rutgers University, 607 Taylor Road, Piscataway, New Jersey 08854, \\ USA \\ ${ }^{2}$ Department of Materials Science and Engineering, National Taiwan University, Taipei 10617, Taiwan
}

(Received 5 January 2008; accepted 9 May 2008; published online 13 June 2008)

\begin{abstract}
The deposition and optoelectronic properties of reduced graphene oxide thin films are described. Thin films with thicknesses ranging from 1-10 $\mathrm{nm}$ have been deposited by the vacuum filtration method. The conductivity of the thin films can be varied over six orders of magnitude by varying the filtration volume of the graphene oxide aqueous suspension while maintaining the transmittance between 60\%-95\%. In addition, enhancement in the conductance through $\mathrm{Cl}$ doping is demonstrated. The combination of the reduction and $\mathrm{Cl}$ treatments make the reduced graphene oxide thin films sufficiently conducting to incorporate them as the hole collecting electrode in proof of concept organic photovoltaic devices. (C) 2008 American Institute of Physics.
\end{abstract}

[DOI: $10.1063 / 1.2937846]$

Interest in the unique properties of graphene has led to numerous studies ${ }^{1-7}$ because it can be easily deposited by the Scotch tape method ${ }^{2}$ and identified on $300 \mathrm{~nm} \mathrm{SiO}_{2}{ }^{3}$ An ideal monolayer of graphene has transparency of $98 \%$ (Ref. 8) with sheet resistance of $\sim 6 \mathrm{k} \Omega / \square$, making it suitable for transparent and conducting electrodes. However, for technological feasibility, uniform deposition over large areas is necessary.

Chemical routes for obtaining single sheets of graphene from reduction of graphene oxide (GO) have been reported. ${ }^{9,10}$ This is appealing because GO can be easily suspended in aqueous suspensions ${ }^{11}$ and deposited on a wide range of substrates.

Although the overall efficiency of the best OPVs is approximately $5 \%,{ }^{13,14}$ lower than inorganics, ${ }^{15}$ the economics could allow their widespread use. Presently, state-of-the-art transparent and conducting hole collecting electrode is indium tin oxide (ITO) (transparency $>90 \%$ at $\lambda=550 \mathrm{~nm}$ and low sheet resistance $=10-30 \Omega / \square)$. However, ITO cracks upon flexing. Thus, to move toward truly flexible OPVs, alternatives to ITO and Al electrodes are needed.

The use of solution deposited single walled carbon nanotube (SWNT) thin films as cathodes in OPVs has been demonstrated. ${ }^{16,17}$ Transparent and conducting thin films from thermal reduction of GO (sheet resistance as low as $\sim 600 \Omega / \square$ with transparency of $\sim 60 \%$ ) at $1100{ }^{\circ} \mathrm{C}$ in $\mathrm{Ar} / \mathrm{H}_{2}$ atmosphere have been reported ${ }^{18,19}$ and demonstrated to be suitable as electrodes for dye sensitized solar cells. ${ }^{18}$ These interesting properties make reduced GO thin films an emerging material for organic electronics.

The transparent and conducting reduced GO thin films were prepared from an aqueous suspension obtained by the exfoliation of graphite oxide using the modified Hummers method. ${ }^{20}$ The structure of GO has been widely studied and consists of the attachment of hydroxyl, carboxyl, and epoxide groups $^{21,22}$ which render it highly soluble in water, allowing exfoliation into individual sheets. We employed the

\footnotetext{
${ }^{\text {a) }}$ Authors to whom correspondence should be addressed. Electronic ad-
} dresses: manish1@rci.rutgers.edu and chunwei@ntu.edu.tw. vacuum filtration technique to deposit uniform layers of GO. ${ }^{12,22-25}$ The technique involves filtrating the suspension containing the GO sheets through a $25 \mathrm{~nm}$ pore size mixed cellulose ester membrane. As the liquid is filtrated, the GO sheets block some of the pores where the permeation rate of the liquid is dramatically reduced. Therefore, in regions where the pores are not blocked, the permeation rate is enhanced until covered by GO sheets. Once the GO films are deposited on the ester membrane, it is necessary to transfer them onto substrates. The transfer process is performed by placing the ester membrane with the film side down onto the substrate. The ester membrane is then etched away by sequential acetone washes. The process is highly repeatable and the films are well adhered to glass and plastic substrates, allowing lithographic processing without delamination. ${ }^{12}$

Scanning electron microscopy (SEM) images of reduced GO thin films on $\mathrm{SiO}_{2} / \mathrm{Si}$ substrate are shown in Figs. 1(a)-1(d). The lighter regions in the SEM images represent either single layer GO or voids between the GO sheets. ${ }^{12}$ It can be seen that the fraction of voids decreases with filtration volume until around $80 \mathrm{~mL}$. The average thickness of the films can be varied from 1 to $5 \mathrm{~nm}$ by varying the filtration volume from 20 to $80 \mathrm{ml}$ while keeping the concentration of GO constant $(0.33 \mathrm{mg} / \mathrm{l})$. A thickness of $1 \mathrm{~nm}$ correlates to a single GO sheet due to functional groups and adsorbed water. ${ }^{26}$ The thicknesses of our thin films have been measured by Raman mapping of 2D peak, ${ }^{27}$ ellipsometry, and atomic force microscope (AFM) profilometry. Raman mapping and ellipsometry results have been reported elsewhere. ${ }^{12}$ The thicknesses versus effective filtration volumes measured by AFM are shown in Fig. 1(e). We define effective filtration volume as the volume of $0.33 \mathrm{mg} / 1$ suspension required to deposit equivalent amount of GO when the concentration of the suspension is changed. Above filtration volumes of $80 \mathrm{ml}$, the film thickness increases almost linearly whereas no significant change is seen for 60 and $70 \mathrm{ml}$. This is attributed to the fact that voids are present at low filtration volumes and a continuous film is achieved at approximately $80 \mathrm{ml}$. 

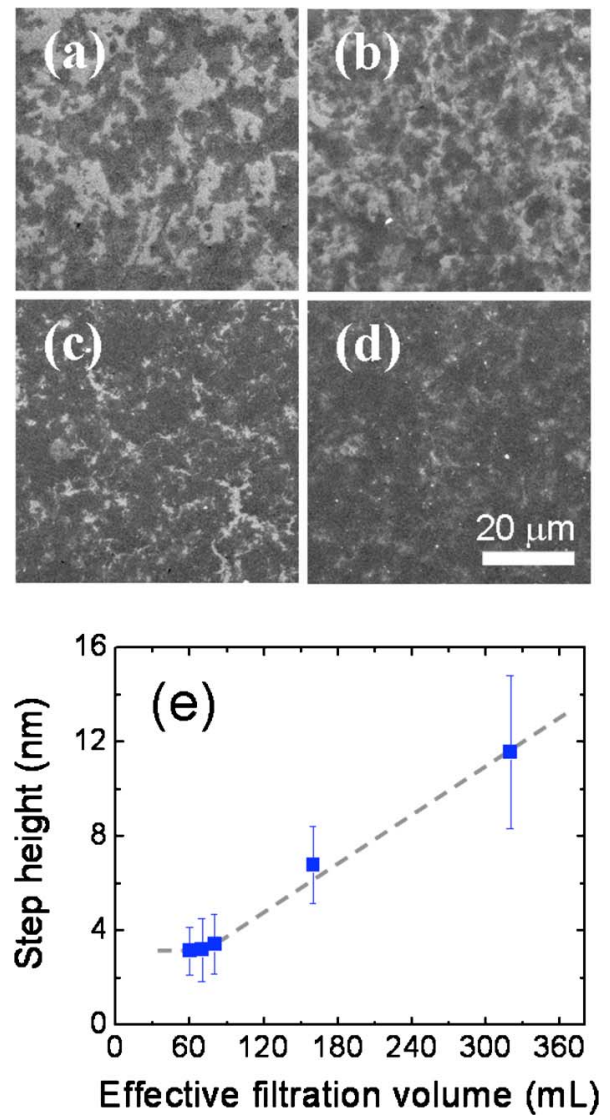

FIG. 1. (Color online) SEM micrographs of reduced GO thin films prepared by filtrating $0.33 \mathrm{mg} / 1 \mathrm{GO}$ suspension in volumes of (a) $20 \mathrm{ml}$, (b) $30 \mathrm{ml}$, (c) $50 \mathrm{ml}$, and (d) $70 \mathrm{ml}$. Contrast is observed between the $\mathrm{SiO}_{2} / \mathrm{Si}$ substrate (light) and reduced GO (dark). The scale bar corresponds to $20 \mu \mathrm{m}$. (e) Reduced GO film thicknesses as a function of effective filtration volume. Films were prepared on $\mathrm{SiO}_{2} / \mathrm{Si}$ substrates and the step height at the edges of a scratch was measured with AFM at more than ten different locations for each sample.

GO thin films are electrically insulating and must be reduced in order to make them conducting. The reduction of GO films can be performed by exposing to hydrazine vapor at room temperature ${ }^{28}$ or at slightly elevated temperatures $\left(80^{\circ} \mathrm{C}\right) .{ }^{9,10}$ The reduction process is believed to occur by breaking the $s p^{3}$ carbon-oxygen bonds which leads to $s p^{2}$ hybridized carbon, thereby recovering $\pi$ electrons. ${ }^{21}$ Elimination of the $s p^{3}$ bonds leads to a higher concentration of delocalized $\pi$ electrons which results in the decrease of resistivity. The reduction treatment has been monitored by x-ray photoelectron spectroscopy, ${ }^{12}$ which shows that the $\mathrm{C}-\mathrm{O}$ bonds are removed with the reduction treatment.

We recently showed ${ }^{12}$ that a combination of hydrazine vapor and low temperature annealing $\left(200^{\circ} \mathrm{C}\right)$ in nitrogen or vacuum reduces the sheet resistance significantly. Figure 2(a) shows sheet resistance of GO films after the combined reduction steps as a function of filtration volume. Figure 2(a) is data from our previous study ${ }^{12}$ but is repeated here for the sake of completion. The sheet resistance decreases dramatically with the filtration volume after annealing but saturates above a critical volume because the reduction treatment is only effective for the top few layers. The transmittance at $550 \mathrm{~nm}$ versus the sheet resistance of the thin films is shown in Fig. 2(b) and was found to monotonously increase in $300-900 \mathrm{~nm}$ wavelength range (not shown) with no clear absorption peaks. It can be seen that the for the lowest sheet Downloaded 16 Jun 2008 to 140.112 .32 .21 . Redistribution subject
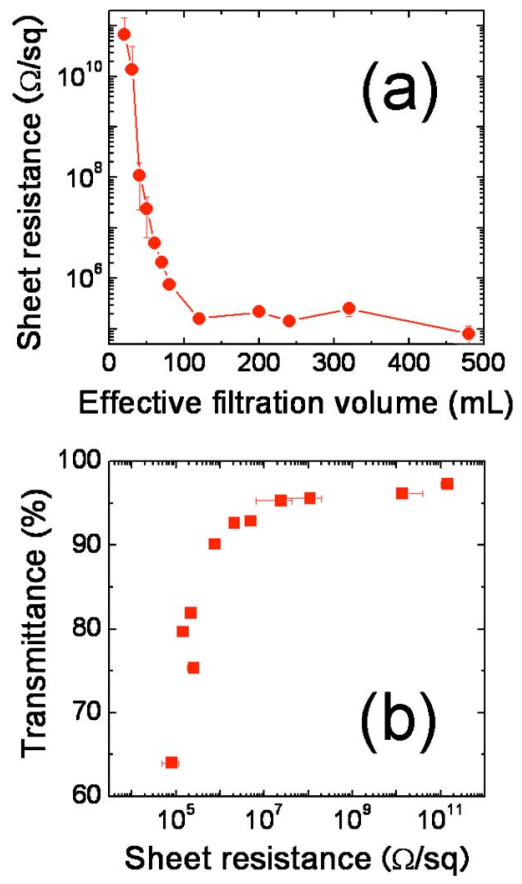

FIG. 2. (Color online) (a) Sheet resistance of reduced GO thin films as a function of effective filtration volume. Measurements were made at more than five different areas of the films. (b) Transmittance at $\lambda=550 \mathrm{~nm}$ as a function of sheet resistance for reduced GO thin films.

resistance $(70 \mathrm{k} \Omega / \square)$, the transmittance of the film is approximately $65 \%$.

In order to further decrease the sheet resistance, we attempted to dope the films by dipping in thionyl chloride $\left(\mathrm{SOCl}_{2}\right)$ for $1 \mathrm{~h}$. We previously demonstrated in SWNT thin films that the attachment of $\mathrm{Cl}$ using this method leads to $p$-type doping and enhanced sheet conductance. ${ }^{29}$ We found that for thin films of reduced GO, the sheet resistance can be reduced by a factor of nearly 5 while for thicker films the effect is moderate. The increase in conductance with $\mathrm{SOCl}_{2}$ treatment is demonstrated in the transfer characteristics of doped and undoped reduced GO thin film transistors (TFTs) shown in Fig. 3. The TFT characteristics are typical of reduced GO devices measured in air. ${ }^{9,10}$ It can be seen that exposure to $\mathrm{SOCl}_{2}$ leads to a fivefold increase in the sourcedrain current which can be translated to a similar decrease in the sheet resistance, as reported in Ref. 12. The neutrality

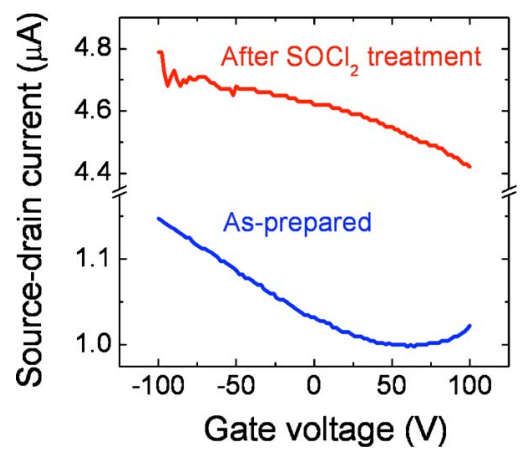

FIG. 3. (Color online) (a) Transfer characteristics of a reduced GO thin film before and after dipping in $\mathrm{SOCl}_{2}$ for $1 \mathrm{~h}$. The film was prepared at a filtration volume of $30 \mathrm{ml}$ with $0.33 \mathrm{mg} / 1 \mathrm{GO}$ suspension and transferred onto a Si substrate with $300 \mathrm{~nm}$ thermally grown oxide. Au was used as source and drain electrodes and the channel length was $21 \mu \mathrm{m}$. The gate

voltage was swept from positive to negative with $1 \mathrm{~V}$ step. 


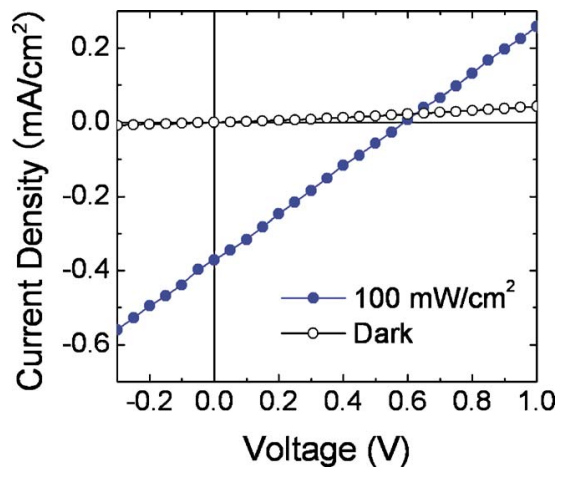

FIG. 4. (Color online) Solar cell characteristics of an OPV with a reduced GO thin film as the transparent electrode. A Newport AM1.5 solar simulator was used to evaluate the photovoltaic characteristics. The film used for the fabrication of OPV had thickness of $\sim 14 \mathrm{~nm}$, sheet resistance of $\sim 40 \mathrm{k} \Omega / \square$, and transmittance of $64 \%$.

point which is observed at the gate voltage of $\sim 60 \mathrm{~V}$ for untreated film moves further toward the positive gate voltage $(>100 \mathrm{~V}$, which is beyond the capability of our measurement apparatus) upon $\mathrm{SOCl}_{2}$ treatment indicating $p$-type doping with $\mathrm{Cl}$.

Proof of concept devices consisting of the reduced and doped GO thin films as the cathodes in OPVs were fabricated. ${ }^{12}$ Here, we provide more details regarding device fabrication and performance. In order to utilize the most conducting material as the electrode, reduced thin films deposited at a filtration volume of $480 \mathrm{ml}$ and treated with $\mathrm{SOCl}_{2}$ by dipping in solution for $1 \mathrm{~h}$ were fabricated. OPV devices were prepared by initially spin coating $(4500 \mathrm{rpm}) \mathrm{a}$ layer of poly(3,4-ethylene dioxythiophene):poly(styrenesulfonate) (PEDOT:PSS) on top of the reduced and doped GO thin films. Subsequently, regioregular (94\%) poly(3hexylthiophene) (P3HT) (molecular weight $~ 58000)$ and phenyl-C61-butyric acid methyl ester (PCBM) nanocomposite layer was deposited on top. The P3HT:PCBM ratio was $1: 1$ and the concentration of solid to chlorobenzene solvent was $20 \mathrm{mg} / \mathrm{ml}$. The spin coating speed for P3HT:PCBM was $700 \mathrm{rpm}$ to achieve a thickness of approximately $120 \mathrm{~nm}$. Thermally evaporated Al was used as the top electrode for the OPV devices.

The current density versus the applied voltage results from the reduced GO electrode devices in dark and under illumination are shown in Fig. 4. The efficiency of the devices was approximately $0.1 \%$. We verified that the output in Fig. 4 was not the result of PEDOT:PSS by fabricating devices where PEDOT:PSS was used as the hole collecting electrode. In all of our devices fabricated with PEDOT:PSS alone, we were not able to make working devices. Thus, the reduced GO in our OPV devices indeed act as hole collecting electrodes. The data under illumination are far from the ideal diode characteristics and the devices are limited by the large resistance (on the order of $10^{5} \Omega / \square$ ) of the reduced GO electrodes. However, the decrease in the sheet resistance of reduced GO should lead to a significant improvement in the OPV device performance.

In conclusion, uniform deposition of transparent and conducting reduced GO thin films is described. The conductance of the thin films was $\sim 10^{5} \Omega / \square$ at a transmittance of $\sim 65 \%$. We also demonstrated that thionyl chloride $\left(\mathrm{SOCl}_{2}\right)$ treatment reduces the sheet resistance by a factor of up to 5 . The $\mathrm{Cl}$ doping allows the utilization of the transparent reduced GO thin films as the hole collecting electrode in organic photovoltaic devices. Thus, our work provides a process path for the incorporation of graphene in organic electronics.

${ }^{1}$ A. K. Geim and K. S. Novoselov, Nat. Mater. 6, 183 (2007).

${ }^{2}$ K. S. Novoselov, A. K. Geim, S. V. Morozov, D. Jiang, Y. Zhang, S. V. Dubonos, I. V. Grigorieva, and A. A. Firsov, Science 306, 666 (2004).

${ }^{3}$ P. Blake, E. W. Hill, A. H. C. Neto, K. S. Novoselov, D. Jiang, R. Yang, T. J. Booth, and A. K. Geim, Appl. Phys. Lett. 91, 063124 (2007).

${ }^{4}$ Y. B. Zhang, Y. W. Tan, H. L. Stormer, and P. Kim, Nature (London) 438, 201 (2005).

${ }^{5}$ K. S. Novoselov, Z. Jiang, Y. Zhang, S. V. Morozov, H. L. Stormer, U. Zeitler, J. C. Maan, G. S. Boebinger, P. Kim, and A. K. Geim, Science 315, 1379 (2007).

${ }^{6}$ S. Pisana, M. Lazzeri, C. Casiraghi, K. S. Novoselov, A. K. Geim, A. C. Ferrari, and F. Mauri, Nat. Mater. 6, 198 (2007).

${ }^{7}$ N. Tombros, C. Jozsa, M. Popinciuc, H. T. Jonkman, and B. J. van Wees, Nature (London) 448, 571 (2007).

${ }^{8}$ R. R. Nair, P. Blake, A. N. Grigorenko, K. Novoselov, T. J. Booth, T. Stauber, N. M. R. Peres, and A. K. Geim, Science (to be published), doi:10.1126/science. 1156965.

${ }^{9}$ S. Gijie, S. Han, M. Wang, K. L. Wang, and R. B. Kaner, Nano Lett. 7, 3394 (2007).

${ }^{10}$ C. Gomez-Navarro, T. R. Weitz, A. M. Bittner, M. Scolari, A. Mews, M. Burghard, and K. Kern, Nano Lett. 7, 3499 (2007).

${ }^{11}$ S. Stankovich, R. D. Piner, X. Q. Chen, N. Q. Wu, S. T. Nguyen, and R. S. Ruoff, J. Mater. Chem. 16, 155 (2006).

${ }^{12}$ G. Eda, G. Fanchini, and M. Chhowalla, Nat. Nanotechnol. 3, 270 (2008).

${ }^{13}$ G. Li, V. Shrotriya, J. S. Huang, Y. Yao, T. Moriarty, K. Emery, and Y. Yang, Nat. Mater. 4, 864 (2005).

${ }^{14}$ M. Reyes-Reyes, K. Kim, and D. L. Carroll, Appl. Phys. Lett. 87, 083506 (2005).

${ }^{15}$ R. W. Miles, G. Zoppi, and I. Forbes, Mater. Today 10, 20 (2007).

${ }^{16}$ A. D. Pasquier, H. E. Unalan, A. Kanwal, S. Miller, and M. Chhowalla, Appl. Phys. Lett. 87, 203511 (2005).

${ }^{17}$ M. W. Rowell, M. A. Topinka, M. D. McGehee, H. J. Prall, G. Dennler, N. S. Sariciftci, L. B. Hu, and G. Gruner, Appl. Phys. Lett. 88, 233506 (2006).

${ }^{18}$ X. Wang, L. Zhi, and K. Mullen, Nano Lett. 8, 323 (2007).

${ }^{19}$ H. A. Becerill, J. Mao, Z. Liu, R. M. Stoltenberg, Z. Bao, and Y. Chen, ACS Nano 2, 463 (2008).

${ }^{20}$ M. Hirata, T. Gotou, S. Horiuchi, M. Fujiwara, and M. Ohba, Carbon 42, 2929 (2004)

${ }^{21}$ S. Stankovich, D. A. Dikin, R. D. Piner, K. A. Kohlhaas, A. Kleinhammes, Y. Jia, Y. Wu, S. T. Nguyen, and R. S. Ruoff, Carbon 45, 1558 (2007).

${ }^{22}$ M. J. McAllister, J. L. Lio, D. H. Adamson, H. C. Schniepp, A. A. Abdala, J. Liu, M. Herrera-Alonso, D. L. Milius, R. Caro, R. K. Prud'homme, and I. A. Aksay, Chem. Mater. 19, 4396 (2007).

${ }^{23}$ H. E. Unalan, G. Fanchini, A. Kanwal, A. Du Pasquier, and M. Chhowalla, Nano Lett. 6, 677 (2006).

${ }^{24}$ Z. C. Wu, Z. H. Chen, X. Du, J. M. Logan, J. Sippel, M. Nikolou, K. Kamaras, J. R. Reynolds, D. B. Tanner, A. F. Hebard, and A. G. Rinzler, Science 305, 1273 (2004)

${ }^{25}$ L. Hu, D. S. Hecht, and G. Gruner, Nano Lett. 4, 2513 (2004).

${ }^{26}$ I. Jung, M. Pelton, R. Piner, D. A. Dikin, S. Stankovich, S. Watcharotone, M. Hausner, and R. S. Ruoff, Nano Lett. 7, 3569 (2007).

${ }^{27}$ A. C. Ferrari, J. C. Meyer, V. Scardaci, C. Casiraghi, M. Lazzeri, F. Mauri, S. Piscanec, D. Jiang, K. S. Novoselov, S. Roth, and A. K. Geim, Phys. Rev. Lett. 97, 187401 (2006).

${ }^{28}$ S. Watcharotone, D. A. Dikin, S. Stankovich, R. Piner, I. Jung, G. H. B. Dommett, G. Evmenenko, S. E. Wu, S. F. Chen, C. P. Liu, S. T. Nguyen, and R. S. Ruoff, Nano Lett. 7, 1888 (2007).

${ }^{29}$ B. B. Parekh, G. Fanchini, G. Eda, and M. Chhowalla, Appl. Phys. Lett. 90, 121913 (2007). 